\title{
THE COST OF COMPUTING INTEGERS
}

\author{
W. DE MELO AND B. F. SVAITER \\ (Communicated by William W. Adams)
}

\begin{abstract}
We analyse the growth rate of a number theoretic function related to the operational complexity of integers
\end{abstract}

The purpose of this note is to answer a question raised by Smale on the cost of computing integers using arithmetic operations. More precisely, let $\tau: \mathbf{N} \rightarrow \mathbf{N}$ be the function that associates to each number $n$ the minimum number of arithmetic operations (addition, subtraction and multiplication) one needs to obtain $n$ starting from 1 and 2. Although 2 is obtainable from 1 in one operation, we have included it as a "starting number" (like 1) to simplify our formulas and induction.

Definition. An allowable list of length $k$ is a list of $k$ integers $n_{1}, n_{2}, \ldots, n_{k}$ such that for each $l \leq k$, there exist integers $-1 \leq i, j<l$ such that $n_{l}=o p\left(n_{i}, n_{j}\right)$, where $o p$ is either addition, subtraction or multiplication and $n_{-1}=1, n_{0}=2$.

It follows that $\tau(n) \leq k$ if and only if there exists an allowable list of length $k$, $\left\{n_{1}, \ldots, n_{k}\right\}$ with $n_{k}=n$. Also, $\tau(n)=k$ if $\tau(n) \leq k$ but $\tau(n)$ is not less than or equal to $k-1$.

Proposition 1. (a) $\log \log (n) \leq \tau(n) \leq 2 \log (n)$, where $\log$ is the logarithm in base 2 .

(b) $\tau\left(2^{2^{k}}\right)=k=\log \left(\log \left(2^{2^{k}}\right)\right)$.

Proof. Suppose that $\tau(n)=k$. Then there exists an allowable list $\left\{n_{1}, \ldots, n_{k}\right\}$ with $n_{k}=n$. Let us consider the allowable list $\left\{m_{1}, \ldots, m_{k}\right\}$, where $m_{l}=m_{l-1} \times m_{l-1}$. By induction we have that $n_{l} \leq m_{l}$ for every $l \leq k$ because $m_{i} \leq m_{j}$ for $i \leq j$. Therefore, $n \leq m_{k}=2^{2^{k}}$. Thus, $\log (\log (n)) \leq k=\tau(n)$. This proves (b) and the first inequality in (a). To prove the second inequality we consider the binary expansion $n=2^{k_{1}}+2^{k_{2}}+\cdots+2^{k_{l}}$, with $0 \leq k_{1}<\cdots<k_{l}$. The following is an allowable sequence:

$$
\left\{2^{2}, 2^{3}, \ldots, 2^{k_{l}}, 2^{k_{l}}+2^{k_{l-1}}, \ldots, 2^{k_{l}}+\cdots+2^{k_{1}}=n\right\} .
$$

Hence, $\tau(n) \leq k_{l}+l \leq 2 \log (n)$.

Remark. $\tau\left(2^{n}\right) \leq 2 \log \log \left(2^{n}\right)$. In fact, if $n=2^{k_{1}}+\cdots+2^{k_{l}}$, then

$$
\left\{2,2^{2}, 2^{2^{2}}, \ldots, 2^{2^{k_{l}}}, 2^{2^{k_{l}}} \times 2^{2^{k_{l}-1}}, \ldots, 2^{2^{k_{l}}+\cdots+2^{k_{1}}}=n\right\}
$$

is an allowable list and, therefore, $\tau(n) \leq k_{l}+l \leq 2 \log \log \left(2^{n}\right)$.

Received by the editors May 31, 1994 and, in revised form, October 24, 1994.

1991 Mathematics Subject Classification. Primary 11N56, 11A25, 11 Y16. 
Lemma 1. Let $B(k)=\{n \in \mathbf{N} ; \tau(n) \leq k\}$. Then the cardinality $\# B(k) \leq 3^{k} \times$ $((k+1) !)^{2}$.

Proof. Let us consider the space $\mathcal{S}_{k}=\left\{s=\left(s_{1}, \ldots, s_{k}\right)\right\}$, where each $s_{l}=\left(\right.$ op $\left._{l}, i_{l}, j_{l}\right)$ and $o p_{l} \in\{+, \times-\}, i_{l}, j_{l}$ are integers smaller than $l$. To each point $s \in \mathcal{S}_{k}$ we can associate an allowable sequence $n_{1}, \ldots, n_{k}$ by taking $n_{l}=o p_{l}\left(n_{i_{l}}, n_{j_{l}}\right)$, starting with $n_{-1}=1$ and $n_{0}=2$. In particular we have a mapping $\phi: \mathcal{S}_{k} \rightarrow B(k)$ which associates to $s$ the integer $n_{k}$ constructed above. Since $\phi$ is onto, it follows that the cardinality of $B(k)$ is at most equal to the cardinality of $\mathcal{S}_{k}$ which is equal to $3^{k} \times((k+1) !)^{2}$.

Definition. A property $P$ holds for almost all integers if the number of integers smaller than $n$ that do not satisfy $P$ is $n \times o(n)$.

Theorem. If $\epsilon>0$, then almost all integers $n$ satisfy the property:

$$
\tau(n) \geq \frac{\log (n)}{(\log \log (n))^{1+\epsilon}} .
$$

Proof. Suppose, by contradiction, that this is not true. Let

$$
\psi(n)=\frac{\log (n)}{(\log \log (n))^{1+\epsilon}} .
$$

Then, there exists $0<\rho<1$ such that, for infinitely many values of $m$, the cardinality of the set

$$
C_{m}=\{n \leq m ; \tau(n) \leq \psi(n)\}
$$

is bigger than $\rho \times m$. If $\psi(m) \leq k<\psi(m)+1$, then $C_{m} \subset B_{k}$. Therefore, by the lemma, $\rho \times m \leq 3^{k}((k+1) \text { ! })^{2}$ for infinitely many values of $m$. Thus,

$$
\rho \times m \leq 3^{\psi(m)+1}(\psi(m)+2)^{2(\psi(m)+2)}
$$

which is a contradiction because a straightforward calculation shows that the above inequality cannot hold for $m$ big enough.

The above theorem answers negatively Smale's first question: does there exist a polynomial $p$ such that $\tau(n) \leq p(\log \log (n))$ ?

Smales's question 2. Is $\tau(k !) \leq p(\log k)$ for some universal polynomial $p$ ?

Smale's question 3. Does there exist a polynomial $p$ such that for each $k$ there exists an $m$ satisfying $\tau(m \times k !) \leq p(\log k)$ ? In [SS], Shub and Smale proved that a negative answer to this question implies that one cannot find an algorithm having polynomial cost to decide whether a family of polynomials have a common zero, and, by the results of [BSS], this implies that $N \neq N P$ over the complex numbers.

\section{REFERENCES}

[SS] M. Shub and S. Smale, On the Intractability of Hilbert's Nullestellensatz and an algebraic version of "NP$\neq P$ ?", preprint.

[BSS] L. Blum, M. Shub, and S. Smale, On a theory of computation and complexity over the real numbers: NP-completeness, recursive functions and universal machines, Bull. Amer. Math. Soc. 21 (1989), 1-46. MR 90a:68022

Instituto de Matematica Pura e Aplicada, Estrada Dona Castorina 110, Jardim Botanico, Rio de Janeiro, BraziL

E-mail address: demelo@impa.br

E-mail address: benar@impa.br 